\title{
Existence and nonexistence of solutions for a generalized Boussinesq equation
}

Ying Wang*

\section{"Correspondence:} nadine_1979@163.com

School of Mathematical Sciences, University of Electronic Science and Technology of China, Chengdu, 611731, China

\begin{abstract}
The Cauchy problem for a generalized Boussinesq equation is investigated. The existence and uniqueness for the local solution and global solution of the problem are established under certain conditions. Moreover, the potential well method is used to discuss the finite-time blow-up for the problem.
\end{abstract}

MSC: 35Q20; 76B15

Keywords: Boussinesq equation; blow-up; global solution; nonexistence

\section{Introduction}

In 1872, the Boussinesq equation was derived by Boussinesq [1] to describe the propagation of small amplitude long waves on the surface of shallow water. This was also the first to give a scientific explanation of the existence to solitary waves. One of the classical Boussinesq equations takes the form

$$
u_{t t}=-a u_{x x x x}+u_{x x}+\beta\left(u^{2}\right)_{x x},
$$

where $u(t, x)$ is an elevation of the free surface of fluid, and the constant coefficients $a$ and $\beta$ depend on the depth of fluid and the characteristic speed of long waves. Extensive research has been carried out to study the classical Boussinesq equation in various respects. The Cauchy problem of (1) has been discussed in [2-10]. In [11-13], the initial boundary value problem and the Cauchy problem for the Boussinesq equation

$$
u_{t t}=u_{x x}+u_{x x t t}+u_{x x x x}-k u_{x x t}+f(u)_{x x}
$$

were studied.

In order to discuss the water wave problem with surface tension, Schneider and Eugene [14] investigated the following Boussinesq model:

$$
u_{t t}=u_{x x}+u_{x x t t}+\mu u_{x x x x}-u_{x x x x t t}+f(u)_{x x}
$$

where $t, x, \mu \in R$ and $u(t, x) \in R$. Equation (3) can also be derived from the 2D water wave problem. For a degenerate case, Schneider and Eugene [14] have proved that the long wave limit can be described approximately by two decoupled Kawahara equations. In $[15,16]$, Wang and Mu studied the well-posedness of the local and globally solution, the blow-up 
of solutions and nonlinear scattering for small amplitude solutions to the Cauchy problem of (3). In $[17,18]$, the authors investigated the Cauchy problem of the following Rosenau equation:

$$
u_{t t}-u_{x x}+u_{x x x x}+u_{x x x x t t}=f(u)_{x x} .
$$

The existence and uniqueness of the global solution and blow-up of the solution for (4) are proved by Wang and Xu [17]. Wang and Wang [18] also proved the global existence and asymptotic behavior of the solution in $n$-dimensional Sobolev spaces. Recently, $\mathrm{Xu}$ et al. $[19,20]$ proved the global existence and finite-time blow-up of the solutions for (4) by means of the family of potential wells. The results in [20] improve the results obtained by Wang and $\mathrm{Xu}$ [17].

This work considers the Cauchy problem for the following equation:

$$
\left\{\begin{array}{l}
u_{t t}-\Delta u_{t t}+\Delta^{2} u_{t t}=-\Delta^{2} u+\Delta u+\Delta f(u), \quad x \in R^{n}, t>0 \\
u(x, 0)=\phi(x), \quad u_{t}(x, 0)=\psi(x), \quad x \in R^{n}
\end{array}\right.
$$

where $f(u)$ satisfies one of the following three assumptions:

$$
\begin{aligned}
& \left(\mathrm{A}_{1}\right) f(u)= \pm a|u|^{p} \quad \text { or } \quad-a|u|^{p-1} u, \quad a>0, p>1, \\
& \left(\mathrm{~A}_{2}\right)\left\{\begin{array}{l}
f(u)= \pm a|u|^{p}, \quad a>0, p>1, p \neq 2 k, k=1,2, \ldots \quad \text { or } \\
f(u)=-a|u|^{p-1} u, \quad a>0, p>1, p \neq 2 k+1, k=1,2, \ldots,
\end{array}\right. \\
& \left(\mathrm{A}_{3}\right)\left\{\begin{array}{l}
f(u)= \pm a|u|^{2 k}, \quad a>0, p>1, k=1,2, \ldots \text { or } \\
f(u)=-a|u|^{2 k+1} u, \quad k=1,2, \ldots
\end{array}\right.
\end{aligned}
$$

In this paper, we discuss problem (5) in high dimensional space. To our knowledge, there have been few results on the global existence of a solution to problem (5). In [21], Wang and Xue only proved the global existence and finite-time blow-up of the solution to (3) in one space dimension. Though the arguments and methods used in this paper are similar to those in [20], the first equation of problem (5) is different from (3) and (4).

By the Fourier transform and Duhamel?s principle, the solution $u$ of problem (5) can be written as

$$
u(t, x)=\left(\partial_{t} S(t) \phi\right)(x)+(S(t) \psi)(x)+\int_{0}^{t} \Gamma(t-\tau) f(u(\tau)) d \tau .
$$

Here $\Gamma(t)=S(t)\left(1-\Delta+\Delta^{2}\right)^{-1} \Delta$ and

$$
\begin{aligned}
& \left(\partial_{t} S(t) \phi\right)(x)=\frac{1}{(2 \pi)^{n}} \int_{R^{n}} e^{i x \xi} \cos \left(\frac{|\xi| \sqrt{1+|\xi|^{2}} t}{\sqrt{1+|\xi|^{2}+|\xi|^{4}}}\right) \hat{\phi}(\xi) d \xi \\
& \left(\partial_{t} S(t) \psi\right)(x)=\frac{1}{(2 \pi)^{n}} \int_{R^{n}} e^{i x \xi} \sin \left(\frac{|\xi| \sqrt{1+|\xi|^{2}} t}{\sqrt{1+|\xi|^{2}+|\xi|^{4}}}\right) \frac{\sqrt{1+|\xi|^{2}+|\xi|^{4}}}{|\xi| \sqrt{1+|\xi|^{2}}} \hat{\psi}(\xi) d \xi
\end{aligned}
$$

where $\hat{\phi}(\xi)=F(\phi)(\xi)=\int_{R^{n}} e^{-i(x, \xi)} \phi(x) d x$ is the Fourier transform of $\phi(x)$.

Throughout this paper: $L^{p}$ denotes the usual Lebesgue space on $R^{n}$ with norm $\|\cdot\|_{L^{p}}$, $H^{s}$ denotes the usual Sobolev space on $R^{n}$ with norm $\|u\|_{H^{s}}=\left\|(I-\Delta)^{\frac{s}{2}} u\right\|=\left\|\left(1+|\xi|^{2}\right)^{\frac{s}{2}} \hat{u}\right\|$ and $|\xi|=\sqrt{\xi_{1}^{2}+\xi_{2}^{2}+\cdots+\xi_{1}^{2}}$. 
First, by using the contraction mapping theorem, we obtain the following existence and uniqueness of the local solution to problem (5).

Theorem 1.1 Let $s>\frac{n}{2}$ and $f \in C^{m}$ with $m \geq s$ being an integer. Then, for any $\phi \in H^{s}$ and $\psi \in H^{s}$, the Cauchy problem (5) has a unique local solution $u \in C^{1}\left([0, T], H^{s}\right)$. Moreover, if $T_{m}$ is the maximal existence time of $u$, and

$$
\max _{0 \leq t<T_{m}}\left[\|u(t)\|_{H^{s}}+\left\|u_{t}(t)\right\|_{H^{s}}\right]<\infty
$$

then $T_{m}=\infty$.

Theorem 1.2 Let $s>\frac{n}{2}$ and $f \in C^{m}$ with $m \geq s$ being an integer. Assume that $\phi \in H^{s}\left(R^{n}\right)$, $\psi \in H^{s}\left(R^{n}\right)$, and $(-\Delta)^{-\frac{1}{2}} \phi \in L^{2}, F(\phi) \in L^{1}, F(u)=\int_{0}^{t} f(\tau) d \tau$. Then, for the local solution $u$, we have $u \in C^{2}\left([0, T) ; H^{s}\right),(-\Delta)^{-\frac{1}{2}} u_{t} \in C^{1}\left(\left[0, T_{m}\right), L^{2}\right)$, satisfying

$$
\begin{aligned}
E(t) & =\frac{1}{2}\left[\left\|(-\Delta)^{-\frac{1}{2}} u_{t}\right\|^{2}+\left\|\nabla u_{t}\right\|^{2}+\|\nabla u\|^{2}+\|u\|^{2}+\left\|u_{t}\right\|^{2}\right]+\int_{R^{n}} F(u) d x \\
& =E(0), \quad \forall t \in\left(0, T_{m}\right) .
\end{aligned}
$$

In order to use the potential well method, for $s>\frac{n}{2}(s \geq 1)$ and $u \in X_{s}(T)$, we define

$$
\begin{aligned}
& J(u)=\frac{1}{2}\|u\|_{H^{1}}^{2}+\int_{R^{n}} F(u) d x, \\
& I(u)=\|u\|_{H^{1}}^{2}+\int_{R^{n}} u f(u) d x, \\
& d=\inf _{u \in N} J(u), \quad N=\left\{u \in H^{1} \mid I(u)=0,\|u\|_{H^{1}} \neq 0\right\}, \\
& W_{1}=\left\{u \in H^{1} \mid I(u)>0, J(u)<d\right\} \cup\{0\}, \\
& V_{1}=\left\{u \in H^{1} \mid I(u)<0, J(u)<d\right\}
\end{aligned}
$$

and

$$
\begin{aligned}
& W_{2}=\left\{u \in H^{1} \mid I(u)>0\right\} \cup\{0\}, \\
& V_{2}=\left\{u \in H^{1} \mid I(u)<0\right\} .
\end{aligned}
$$

From $u \in C^{1}\left([0, T] ; H^{s}\right)$, we get $u \in C^{1}\left([0, T] ; L^{\infty}\right)$ and $u \in C^{1}\left([0, T] ; L^{q}\right)$ for all $2 \leq q<\infty$. Hence, $J(u), I(u), d, W_{1}$, and $V_{1}$ are all well defined. Now, we give the following results for problem (5).

Theorem 1.3 Let $s>\frac{n}{2}$ with $s \geq 1$, and $f(u)$ satisfy $\left(\mathrm{A}_{2}\right)$ with $[p] \geq s$ or $\left(\mathrm{A}_{3}\right)$. Assume that $\phi \in H^{s}, \psi \in H^{s}$, and $(-\Delta)^{-\frac{1}{2}} \psi \in L^{2}, E(0)<d$. Then both $W_{2}$ and $V_{2}$ are invariant under the flow of problem (5).

Theorem 1.4 Let $n \leq 3$ and $f(u)$ satisfy $\left(\mathrm{A}_{1}\right)$, where $2 \leq p<\infty$ for $n=1,2 ; \frac{7}{3} \leq p \leq 5$ for $n=3, \phi \in H^{2}, \psi \in H^{2}$, and $(-\Delta)^{-\frac{1}{2}} \phi \in L^{2}$. Assume $E(0)<d$ and $\phi \in W_{2}$. Then problem (5) admits a unique global solution $u \in C^{2}\left([0, \infty), H^{2}\right)$, with $(-\Delta)^{-\frac{1}{2}} u_{t} \in C^{1}\left([0, \infty), L^{2}\right)$ and $u \in W_{1}$ for $0 \leq t<\infty$. 
Theorem 1.5 Let $n \leq 3$ and let $f(u)$ satisfy $\left(\mathrm{A}_{1}\right)$, where $3 \leq p<\infty$ for $n=1,2 ; 3 \leq p \leq 5$ for $n=3, \phi \in H^{3}, \psi \in H^{3}$, and $(-\Delta)^{-\frac{1}{2}} \phi \in L^{2}$. Assume that $E(0)<d$ and $\phi \in W_{2}$. Then problem (5) admits a unique global solution $u \in C^{2}\left([0, \infty), H^{3}\right)$ with $(-\Delta)^{-\frac{1}{2}} u_{t} \in C^{1}\left([0, \infty), L^{2}\right)$ and $u \in W$ for $0 \leq t<\infty$.

Theorem 1.6 Let $n \leq 3$ and $f(u)$ satisfy $\left(\mathrm{A}_{1}\right)$, where $4 \leq p<\infty$ for $n=1,2 ; 4 \leq p \leq 5$ for $n=3, \phi \in H^{4}, \psi \in H^{4}$, and $(-\Delta)^{-\frac{1}{2}} \phi \in L^{2}$. Assume that $E(0)<d$ and $\phi \in W_{2}$. Then problem (5) admits a unique global solution $u \in C^{2}\left([0, \infty), H^{4}\right)$ with $(-\Delta)^{-\frac{1}{2}} u_{t} \in C^{1}\left([0, \infty), L^{2}\right)$ and $u \in W$ for $0 \leq t<\infty$.

Theorem 1.7 Let $s>\frac{n}{2}$ with $s \geq 1$, and $f(u)$ satisfy $\left(\mathrm{A}_{2}\right)$ with $[p] \geq 3$ or $\phi, \psi \in H^{s}$, $(-\Delta)^{-\frac{1}{2}} \phi,(-\Delta)^{-\frac{1}{2}} \psi \in L^{2}$. Assume that $E(0)<d$ and $I(\phi)<0$. Then the solution of problem (5) blows up in finite time, i.e., the maximal existence time $T_{m}$ of $u$ is finite, and

$$
\lim _{t \rightarrow T_{m}} \sup \left(\|u(t)\|_{H^{s}}+\left\|u_{t}(t)\right\|_{H^{s}}\right)=\infty .
$$

The remainder of this paper is organized as follows. In Section 2, Theorems 1.1 and 1.2 are proved. In Section 3, we give some preliminary lemmas and the proof of Theorem 1.3. The proofs of Theorems 1.4, 1.5 and 1.6 are given in Section 4. Finally, Section 5 is devoted to the proof of Theorem 1.7.

\section{Existence of local solutions}

In this section, we consider the local existence and uniqueness of solutions to problem (5).

Lemma 2.1 For the operators $\partial_{t} S(t), S(t)$ and $\Gamma(t)$ defined in Section 1, we have

$$
\begin{aligned}
& \left\|\partial_{t} S(t) \phi\right\|_{H^{s}} \leq\|\phi\|_{H^{s}}, \quad \forall \phi \in H^{s}, \\
& \left\|\partial_{t} S(t) \psi\right\|_{H^{s}} \leq 2(1+t)\|\psi\|_{H^{s}}, \quad \forall \psi \in H^{s}, \\
& \left\|\partial_{t t} S(t) \phi\right\|_{H^{s}} \leq \sqrt{2}\|\phi\|_{H^{s}}, \quad \forall \phi \in H^{s}, \\
& \|\Gamma(t) f\|_{H^{s}} \leq \sqrt{2}\|f\|_{H^{s-2}}, \quad \forall f \in H^{s-2}, \\
& \left\|\partial_{t} \Gamma(t) f\right\|_{H^{s}} \leq\|f\|_{H^{s-2}}, \quad \forall f \in H^{s-2} .
\end{aligned}
$$

Proof We only need to prove (9) and (11), since the proofs of the other inequalities are similar. Using the Plancherel theorem, we have

$$
\begin{aligned}
\left\|\partial_{t} S(t) \psi\right\|_{H^{s}}^{2}= & \int_{R}\left(1+|\xi|^{2}\right)^{s} \frac{1+|\xi|^{2}+|\xi|^{4}}{|\xi|^{2}\left(1+|\xi|^{2}\right)} \sin \left(\frac{t|\xi| \sqrt{1+|\xi|^{2}}}{\sqrt{1+|\xi|^{2}+|\xi|^{4}}}\right)|\hat{\psi}(\xi)|^{2} d \xi \\
\leq & \int_{|\xi| \leq 1}\left(1+|\xi|^{2}\right)^{s} t^{2}|\hat{\psi}(\xi)|^{2} d \xi \\
& +\int_{|\xi|>1}\left(1+|\xi|^{2}\right)^{s} \frac{\left(1+|\xi|^{2}+|\xi|^{4}\right)^{2}}{|\xi|^{2}\left(1+|\xi|^{2}\right)}|\hat{\psi}(\xi)|^{2} d \xi \\
\leq & t^{2} \int_{|\xi| \leq 1}\left(1+|\xi|^{2}\right)^{s}|\hat{\psi}(\xi)|^{2} d \xi+4 \int_{|\xi|>1}\left(1+|\xi|^{2}\right)^{s}|\hat{\psi}(\xi)|^{2} d \xi \\
\leq & 4(1+t)^{2}\|\psi\|_{H^{s}}^{2},
\end{aligned}
$$




$$
\begin{aligned}
\|\Gamma(t) f\|_{H^{s}}^{2}= & \int_{R}\left(1+|\xi|^{2}\right)^{s} \sin ^{2}\left(\frac{t|\xi| \sqrt{1+|\xi|^{2}}}{\sqrt{1+|\xi|^{2}+|\xi|^{4}}}\right) \\
& \times \frac{1+|\xi|^{2}+|\xi|^{4}}{|\xi|^{2}\left(1+|\xi|^{2}\right)} \frac{|\xi|^{4}}{\left(1+|\xi|^{2}+|\xi|^{4}\right)^{2}}|\hat{f}(\xi)|^{2} d \xi \\
\leq & 2 \int_{R}\left(1+|\xi|^{2}\right)^{s-2}|\hat{f}(\xi)|^{2} d \xi=2\|f\|_{H^{s-2}}^{2} .
\end{aligned}
$$

Therefore (9) and (11) hold. This completes the proof of the lemma.

Lemma 2.2 ([17]) Let $g \in C^{m}(R)$, where $m \geq 0$ is an integer.

(i) If $0 \leq s \leq m$ and $u \in H^{s}\left(R^{n}\right) \cap L^{\infty}\left(R^{n}\right)$, then $g(u) \in H^{s}\left(R^{n}\right)$ and

$$
\|g(u)\|_{H^{s}} \leq C\left(\|u\|_{\infty}\right)\|u\|_{H^{s}}
$$

(ii) If $s \leq m$ and $u, v \in H^{s}\left(R^{n}\right) \cap L^{\infty}\left(R^{n}\right)$, then

$$
\|g(u)-g(v)\|_{H^{s}} \leq K\left(\|u\|_{\infty},\|v\|_{\infty}\right)\|u-v\|_{H^{s}}
$$

In particular, if $u, v \in H^{s}$ for some $s>\frac{n}{2}$, then $u$ and $v \in L^{\infty}$, (13) and (14) hold.

Proof of Theorem 1.1 Let $s>\frac{n}{2}$,

$$
\begin{aligned}
& X_{s}(T)=C^{1}\left([0, T] ; H^{s}\right), \\
& \|u\|_{X_{S}(T)}=\max _{0 \leq t \leq T}\left\{\|u(t)\|_{H^{s}}+\left\|u_{t}(t)\right\|_{H^{s}}\right\}
\end{aligned}
$$

and

$$
\begin{aligned}
& B u=\left(\partial_{t} S(t) \phi\right)(x)+(S(t) \psi)(x)+\int_{0}^{t} \Gamma(t-\tau) f(u(\tau)) d \tau, \\
& A_{R}(T)=\left\{u \in X_{S}(T) \mid\|u\|_{X_{S}(T)}<R\right\} .
\end{aligned}
$$

Similarly to the proofs in $[15,16]$, we see that for sufficiently small $T$,

$$
B u: A_{R} \rightarrow A_{R}
$$

is a contract mapping. Hence by the contracting-mapping principle we obtain the result of Theorem 1.1.

Corollary 2.3 Under the assumption of Theorem 1.1, if $T_{m}<\infty$, we have

$$
\lim _{t \rightarrow T_{m}} \sup \left(\|u(t)\|_{H^{s}}+\left\|u_{t}(t)\right\|_{H^{s}}\right)=+\infty
$$

Corollary 2.4 Let $s>\frac{n}{2}$ and $f(u)$ satisfy $\left(\mathrm{A}_{2}\right)$ or $\left(\mathrm{A}_{3}\right)$. Then, for any $\phi \in H^{s}$ and $\psi \in H^{s}$, problem (5) admits a unique local solution $u \in C^{1}\left(\left[0, T_{m}\right), H^{s_{1}}\right)$, where $T_{m}$ is the maximal existence time of $u$. Moreover, either $T_{m}=+\infty$ or $T_{m}<\infty$ and

$$
\lim _{t \rightarrow T_{m}} \sup \left(\|u(t)\|_{H^{s}}+\left\|u_{t}(t)\right\|_{H^{s}}\right)=+\infty
$$


Lemma 2.5 Assume $s>\frac{n}{2}, f \in C^{m}(R), \phi \in H^{s}$, and $\psi \in H^{s}$. Then for the local solution $u \in C^{1}\left(\left[0, T_{m}\right), H^{s}\right)$ given in Theorem 1.1 , we have $u_{t t} \in C\left(\left[0, T_{m}\right), H^{s}\right)$.

Proof Using the Fourier transformation, we have

$$
\hat{u}_{t t}=-\frac{|\xi|^{2}\left(1+|\xi|^{2}\right)}{1+|\xi|^{2}+|\xi|^{4}} \hat{u}+\frac{|\xi|^{2}}{1+|\xi|^{2}+|\xi|^{4}} \hat{f}(u)
$$

Since

$$
\begin{aligned}
& |\xi|^{2}\left(1+|\xi|^{2}\right)<\left(1+|\xi|^{2}+|\xi|^{4}\right), \\
& |\xi|^{2}<\left(1+|\xi|^{2}+|\xi|^{4}\right),
\end{aligned}
$$

which together with (15) yields

$$
\left\|u_{t t}\right\|_{H^{s}}=\left\|\hat{u}_{t t}\right\|_{H^{s}} \leq\|\hat{u}\|_{H^{s}}+\|\hat{f}(u)\|_{H^{s}} \leq\left(1+C\|u\|_{\infty}\right)\|u\|_{H^{s}} \leq C(T) .
$$

Furthermore, using

$$
\begin{aligned}
\left\|u_{t t}(t+\Delta t)-u_{t t}(t)\right\|_{H^{s}} & =\left\|\hat{u}_{t t}(t+\Delta t)-\hat{u}_{t t}(t)\right\|_{H^{s}} \\
& \leq C(T)\|u(t+\Delta t)-u(t)\|_{H^{s}} \rightarrow 0 \quad \text { as } \Delta t \rightarrow 0,
\end{aligned}
$$

we obtain $u_{t t} \in C\left(\left[0, T_{m}\right) ; H^{s}\right)$.

Lemma 2.6 Assume $s>\frac{n}{2}, f \in C^{m}(R), \phi \in H^{s}, \psi \in H^{s}$, and $(-\Delta)^{-\frac{1}{2}} \phi \in L^{2}$. Then for the local solution $u \in C^{1}\left(\left[0, T_{m}\right), H^{s}\right)$ given in Theorem 1.1 , we have $(-\Delta)^{-\frac{1}{2}} u_{t} \in C^{1}\left(\left[0, T_{m}\right), L^{2}\right)$.

Proof First for the local solution $u$ given in Theorem 1.1, we obtain

$$
(-\Delta)^{-\frac{1}{2}} u_{t t} \in C\left(\left[0, T_{m}\right), H^{s+1}\right) .
$$

From (15), we get

$$
\begin{aligned}
& \frac{1}{|\xi|} \hat{u}_{t t}=-\frac{|\xi|^{2}\left(1+|\xi|^{2}\right)}{|\xi|\left(1+|\xi|^{2}+|\xi|^{4}\right)} \hat{u}+\frac{|\xi|^{2}}{|\xi|\left(1+|\xi|^{2}+|\xi|^{4}\right)} \hat{f}(u), \\
& \begin{aligned}
\left\|\frac{|\xi|\left(1+|\xi|^{2}\right)}{1+|\xi|^{2}+|\xi|^{4}} \hat{u}\right\|_{H^{s+1}}^{2} & =\int_{R^{n}}(1+|\xi|)^{s+1} \frac{|\xi|^{2}\left(1+|\xi|^{2}\right)^{2}}{\left(1+|\xi|^{2}+|\xi|^{4}\right)^{2}}|\hat{u}(\xi)|^{2} d \xi \\
\leq & \int_{R^{n}}\left(1+|\xi|^{2}\right)^{s} \frac{\left(1+|\xi|^{2}\right)^{4}}{\left(1+|\xi|^{2}+|\xi|^{4}\right)^{2}}|\hat{u}(\xi)|^{2} d \xi \\
\leq & C \int_{R^{n}}\left(1+|\xi|^{2}\right)^{s}|\hat{u}(\xi)|^{2} d \xi=C\|u\|_{H^{s}}^{2}, \\
& =\int_{R^{n}}(1+|\xi|)^{s+1} \frac{|\xi|^{2}}{\left(1+|\xi|^{2}+|\xi|^{4}\right)^{2}}|\hat{f}(u)|^{2} d \xi \\
& \leq \int_{R^{n}}(1+|\xi|)^{s+1} \frac{\left(1+|\xi|^{2}\right)^{4}}{\left(1+|\xi|^{2}+|\xi|^{4}\right)}|\hat{f}(u)|^{2} d \xi
\end{aligned}
\end{aligned}
$$




$$
\begin{aligned}
& \leq C \int_{R^{n}}\left(1+|\xi|^{2}\right)^{s}|\hat{f}(u)|^{2} d \xi=C\|\hat{f}(u)\|_{H^{s}}^{2} \\
& \leq C\left(\|u\|_{H^{s}}\right)\|u\|_{H^{s}}^{2} .
\end{aligned}
$$

Furthermore, we get $\left\|(-\Delta)^{-\frac{1}{2}} u_{t t}(t+\Delta t)-(-\Delta)^{-\frac{1}{2}} u_{t t}(t)\right\|_{H^{s+1}} \rightarrow 0$ as $\Delta t \rightarrow 0$.

Hence, we have

$$
(-\Delta)^{-\frac{1}{2}} u_{t t} \in C\left(\left[0, T_{m}\right), H^{s+1}\right) .
$$

Using

$$
(-\Delta)^{-\frac{1}{2}} u_{t} \in L^{2}
$$

and

$$
(-\Delta)^{-\frac{1}{2}} u_{t}=(-\Delta)^{-\frac{1}{2}} \psi+\int_{0}^{t}(-\Delta)^{-\frac{1}{2}} u_{\tau \tau} d \tau
$$

we get

$$
(-\Delta)^{-\frac{1}{2}} u_{t} \in C^{1}\left(\left[0, T_{m}\right), L^{2}\right)
$$

Proof of Theorem 1.2 Using (5), it follows by straightforward calculation that

$$
\begin{aligned}
E^{\prime}(t) & =\left((-\Delta)^{-\frac{1}{2}} u_{t t},(-\Delta)^{-\frac{1}{2}} u_{t}\right)+\left(u_{t t}, u_{t}\right)+\left(\nabla u_{t t}, \nabla u_{t}\right)+\left(u_{x}, \nabla u_{t}\right)+\left(u, u_{t}\right)+\left(f(u), u_{t}\right) \\
& =\left\langle(-\Delta)^{-1} u_{t t}+u_{t t}-\Delta u_{t t}-\Delta u+u+f(u), u_{t}\right\rangle_{X * X}=0,
\end{aligned}
$$

where $(\cdot, \cdot)$ denotes the inner product of $L^{2}$ space, $\langle\cdot, \cdot\rangle_{X * X}$ means the usual duality of $X^{*}$ and $X$ with $X=H^{1}$. Integrating the above equality with respect to $t$, we have identity (7). The theorem is proved.

Corollary 2.7 Let $s>\frac{n}{2}$ with $s \geq 1$ and $f(u)$ satisfy $\left(\mathrm{A}_{2}\right)$, with $[p] \geq s$ or $\left(\mathrm{A}_{3}\right)$. Assume that $\phi \in H^{s}, \psi \in H^{s}$, and $(-\Delta)^{-\frac{1}{2}} \psi \in L^{2}$, problem (5) admits a unique local solution $u \in C^{2}\left(\left[0, T_{m}\right), H^{s}\right)$, with $(-\Delta)^{-\frac{1}{2}} u_{t} \in C^{1}\left(\left[0, T_{m}\right), L^{2}\right)$ satisfying $(7)$, where $T_{m}$ is the maximal existence time of $u$. Moreover, either $T_{m}=+\infty$ or $T_{m}<\infty$ and

$$
\lim _{t \rightarrow T_{m}} \sup \left(\|u(t)\|_{H^{s}}+\left\|u_{t}(t)\right\|_{H^{s}}\right)=+\infty
$$

Proof Since $\phi \in H^{s}$ and $s>\frac{n}{2}$, we have $\phi \in L^{\infty}$. Hence, $\phi \in L^{q}$ for all $2 \leq q \leq \infty$. From $|F(\phi)|=C|\phi|^{p+1}, 2<p+1<\infty$. We obtain $F(\phi) \in L^{1}$.

\section{Preliminary lemmas and invariant sets}

In this section, we will prove several lemmas which are related with the potential well for problem (5). By arguments similar to those in [20], we obtain the following lemmas.

Lemma 3.1 Let $s>\frac{n}{2}$ with $s \geq 1$ and let $f(u) \operatorname{satisfy}\left(\mathrm{A}_{1}\right), u \in H^{s}$ and $g(\lambda)=-\frac{1}{\lambda} \int_{R^{n}} u f(\lambda u) d x$. Assume $\int_{R^{n}} u f(u) d x<0$. Then: 
(i) $g(\lambda)$ is increasing on $0<\lambda<\infty$.

(ii) $\lim _{\lambda \rightarrow 0} g(\lambda)=0, \lim _{\lambda \rightarrow+\infty} g(\lambda)=+\infty$.

Lemma 3.2 Let $s>\frac{n}{2}$ with $s \geq 1, u \in H^{s}$, and let $f(u)$ satisfy $\left(\mathrm{A}_{1}\right), u \neq 0$. We have:

(i) $\lim _{\lambda \rightarrow 0} J(\lambda u)=0$.

(ii) $I(\lambda u)=\lambda \frac{d}{d \lambda} J(\lambda u), \forall \lambda>0$.

Furthermore, if $\int_{R^{n}} u f(u) d x<0$, then:

(iii) $\lim _{\lambda \rightarrow+\infty} J(\lambda u)=-\infty$.

(iv) In the interval $0<\lambda<\infty$, there exists a unique $\lambda^{*}=\lambda^{*}(u)$ such that

$$
\left.\frac{d}{d \lambda} J(\lambda u)\right|_{\lambda=\lambda^{*}}=0
$$

(v) $J(\lambda u)$ is increasing on $0<\lambda<\lambda^{*}$, decreasing on $\lambda^{*}<\lambda<\infty$ and $I\left(\lambda^{*}\right)=0$.

(vi) $I(\lambda u)>0$ for $0<\lambda<\lambda^{*}, I(\lambda u)<0$ for $\lambda^{*}<\lambda<\infty$ and $I\left(\lambda^{*}\right)=0$.

Lemma 3.3 Let $s>\frac{n}{2}$ with $s \geq 1, u \in H^{s}$, and let $f(u)$ satisfy $\left(\mathrm{A}_{1}\right)$. Then:

(i) If $0<\|u\|_{H^{1}}<r_{0}$, then $I(u)>0$.

(ii) If I $(u)<0$, then $\|u\|_{H^{1}}>r_{0}$.

(iii) If I $(u)=0$ and $\|u\|_{H^{1}} \neq 0$, then $\|u\|_{H^{1}} \geq r_{0}$, where $r_{0}=\left(\frac{1}{a C_{*}^{p+1}}\right)^{\frac{1}{p-1}}$.

Lemma 3.4 Let $s>\frac{n}{2}$ with $s \geq 1$ and $f(u)$ satisfy $\left(\mathrm{A}_{1}\right)$. We have

$$
d \geq d_{0}=\frac{p-1}{2(p+1)} r_{0}^{2}=\frac{p-1}{2(p+1)}\left(\frac{1}{a C_{*}^{p+1}}\right)^{\frac{2}{p-1}} .
$$

Lemma 3.5 Let $s>\frac{n}{2}$ with $s \geq 1$ and $f(u)$ satisfy $\left(\mathrm{A}_{1}\right)$. Assume $u \in H^{s}$ and $I(u)<0$. Then

$$
d<\frac{p-1}{2(p+1)}\|u\|_{H^{1}}^{2}
$$

Proof of Theorem 1.3 We only prove the invariance of $W_{1}$ since the proof for the invariance of $V_{1}$ is similar. Let $u(t, x)$ be any weak solution of problem (5) with $\phi \in W_{1}, T$ be the maximal existence time of $u(t, x)$. Next we prove that $u(t, x) \in W_{1}$ for $0<t<T$. Arguing by contradiction we assume there is a $t_{1} \in(0, T)$ such that $u\left(t_{1}\right) \notin W_{1}$. By the continuity of $I(u(t))$ with respect to $t$, there exists a $t_{0} \in(0, T)$ such that $u\left(t_{0}\right) \in \partial W_{1}$. From the definition of $W_{1}$ and (i) of Lemma 3.3 we have $R_{0} \subset W_{1}, R_{0}=\left\{u \in H^{1} \mid\|u\|_{H^{1}}<r_{0}\right\}$. Hence we know $0 \notin \partial W_{1}$. From $u\left(t_{0}\right) \in \partial W_{1}$, it holds that $I\left(u\left(t_{0}\right)\right)=0$ and $\left\|u\left(t_{0}\right)\right\|_{H^{1}} \neq 0$. The definition of $d$ yields $J\left(u\left(t_{0}\right)\right) \geq d$, which contradicts

$$
\frac{1}{2}\left[\left\|(-\Delta)^{-\frac{1}{2}} u_{t}\right\|^{2}+\left\|\nabla u_{t}\right\|^{2}+\left\|u_{t}\right\|^{2}\right]+J(u) \leq E(0)<d .
$$

The proof of Theorem 1.3 is complete.

From Theorem 1.3, we can prove the following corollaries.

Corollary 3.6 Let $s, f(u), \phi, \psi$ and $E(0)$ be the same as those in Theorem 1.3. Then:

(i) All solutions of problem (5) belong to $W_{1}$, provided that $\phi \in W_{2}$.

(ii) All solutions of problem (5) belong to $V_{1}$, provided that $\phi \in V_{2}$. 
Corollary 3.7 Let $s>\frac{n}{2}$ with $s \geq 1$, and let $f(u)$ satisfy $\left(\mathrm{A}_{2}\right)$ with $[p] \geq s$ or $\left(\mathrm{A}_{3}\right), \phi \in H^{s}$, $\psi \in H^{s}$ and $(-\Delta)^{-\frac{1}{2}} \psi \in L^{2}$. Assume that $E(0)<0$ or $E(0)=0, \phi \neq 0$. Then all the solutions of problem (5) belong to $V_{1}$.

\section{Global existence of solutions}

In this section, we prove the global existence of a solution for problem (5).

Lemma 4.1 Let $s>\frac{n}{2}$ with $s \geq 1$ and $f(u)$ satisfy $\left(\mathrm{A}_{2}\right)$ with $[p] \geq s$ or $\phi \in H^{s}, \psi \in H^{s}$, and $(-\Delta)^{-\frac{1}{2}} \psi \in C^{1}\left(\left[0, T_{m}\right), L^{2}\right)$. Assume that $E(0)<d$ and $\phi \in W_{2}$. Then, for the local solution u given in Corollary 2.7, one has

$$
\left\|u_{t}(t)\right\|_{H^{1}}^{2}+\|u(t)\|_{H^{1}}^{2}<\frac{4 p}{p-1} d
$$

Proof Let $u$ be the unique local solution of problem (5) given in Corollary 2.7. Then $u \in$ $C^{2}\left(\left[0, T_{m}\right) ; H^{s}\right),(-\Delta)^{-\frac{1}{2}} u_{t} \in C^{1}\left(\left[0, T_{m}\right), L^{2}\right)$ satisfying $(7)$ and

$$
\frac{1}{2}\left\|(-\Delta)^{-\frac{1}{2}} u_{t}\right\|^{2}+\frac{1}{2}\left\|\nabla u_{t}\right\|^{2}+\frac{1}{2}\left\|u_{t}\right\|^{2}+\frac{p-1}{2(p+1)}\|u\|_{H^{1}}^{2}+\frac{1}{p+1} I(u)=E(0)<d .
$$

From Theorem 1.3, we get $u \in W_{2}$ and $I(u) \geq 0$ for $0 \leq t \leq T_{m}$. Hence, (19) gives rise to

$$
\begin{aligned}
& \|u\|_{H^{1}}^{2}<\frac{2(p+1)}{p-1} d, \quad 0 \leq t<T_{m}, \\
& \frac{1}{2}\left\|(-\Delta)^{-\frac{1}{2}} u_{t}\right\|^{2}+\frac{1}{2}\left\|\nabla u_{t}\right\|^{2}+\frac{1}{2}\left\|u_{t}\right\|^{2}<d, \quad 0 \leq t<T_{m} .
\end{aligned}
$$

Thus, we obtain (18).

Proof of Theorem 1.4 It follows from Corollary 2.7 that problem (5) admits a unique local solution $u \in C^{2}\left(\left[0, T_{m}\right) ; H^{2}\right)$, with $(-\Delta)^{-\frac{1}{2}} u_{t} \in C^{1}\left(\left[0, T_{m}\right) ; L^{2}\right)$ satisfying (7), where $T_{m}$ is the maximal existence time of $u$.

Next, we prove that $T_{m}=+\infty$. Using Lemma 4.1 one derives (19). Since $u \in C^{2}\left(\left[0, T_{m}\right)\right.$; $H^{2}$ ) satisfies (5), we have

$$
u_{t t}-\Delta u_{t t}+\Delta^{2} u_{t t}+\Delta^{2} u-\Delta u=\Delta f(u) \quad \text { in } C\left(\left[0, T_{m}\right), H^{-2}\right)
$$

and

$$
u_{t t}+u-\Delta u_{t t}+\Delta^{2} u_{t t}+\Delta^{2} u-\Delta u=\Delta f(u)+u, \quad 0 \leq t<T_{m} .
$$

Multiplying (22) by $u_{t} \in C^{1}\left(\left[0, T_{m}\right), H^{2}\right)$ and integrating on $R^{n}$, we obtain

$$
\begin{aligned}
& \frac{1}{2} \frac{d}{d t}\left[\left\|u_{t}\right\|^{2}+\left\|\nabla u_{t}\right\|^{2}+\left\|\Delta u_{t}\right\|^{2}+\|u\|^{2}+\|\nabla u\|^{2}+\|\Delta u\|^{2}\right] \\
& \quad=-\left(f^{\prime}(u) \nabla u, \nabla u_{t}\right)+\left(u, u_{t}\right) .
\end{aligned}
$$

For $n=3$, we get

$$
-\left(f^{\prime}(u) \nabla u, \nabla u_{t}\right) \leq\left\|f^{\prime}(u)\right\|_{\frac{3}{2}}\|\nabla u\|_{6}\left\|\nabla u_{t}\right\|_{H^{2}}
$$


For $n=3$, we have $H^{1} \hookrightarrow L^{2}$ for $2 \leq q \leq 6,\left|f^{\prime}(u)\right|=A|u|^{p-1}$. From $\frac{7}{3} \leq p \leq 5$, we have $\frac{4}{3} \leq p-1 \leq 4$ and $2 \leq \frac{3}{2}(p-1) \leq 6$. Hence, we have $\left\|f^{\prime}(u)\right\|_{\frac{3}{2}} \leq C(p)$ for $0 \leq t<T_{m}$. From (23) and (24), we get

$$
\begin{aligned}
& \frac{1}{2} \frac{d}{d t}\left[\left\|u_{t}\right\|^{2}+\left\|\nabla u_{t}\right\|^{2}+\left\|\Delta u_{t}\right\|^{2}+\|u\|^{2}+\|\nabla u\|^{2}+\|\Delta u\|^{2}\right] \\
& \quad \leq C\left(\left\|u_{t}\right\|^{2}+\left\|\nabla u_{t}\right\|^{2}+\left\|\Delta u_{t}\right\|^{2}+\|u\|^{2}+\|\nabla u\|^{2}+\|\Delta u\|^{2}\right) .
\end{aligned}
$$

For $n=1$ or 2 , we have

$$
\begin{aligned}
-\left(f^{\prime}(u) \nabla u, \nabla u_{t}\right) & \leq\left\|f^{\prime}(u)\right\|_{3}\|\nabla u\|_{3}\left\|\nabla u_{t}\right\|_{3} \leq C\|u\|_{H^{2}}\left\|u_{t}\right\|_{H^{2}} \\
& \leq C\left(\left\|u_{t}\right\|^{2}+\left\|\nabla u_{t}\right\|^{2}+\left\|\Delta u_{t}\right\|^{2}+\|u\|^{2}+\|\nabla u\|^{2}+\|\Delta u\|^{2}\right)
\end{aligned}
$$

and (25). Let

$$
E_{1}(t)=\frac{1}{2}\left(\left\|u_{t}\right\|^{2}+\left\|\nabla u_{t}\right\|^{2}+\left\|\Delta u_{t}\right\|^{2}+\|u\|^{2}+\|\nabla u\|^{2}+\|\Delta u\|^{2}\right) .
$$

Using (25) yields

$$
E_{1}(t)=E_{1}(0)+C \int_{0}^{t} E_{1}(\tau) d \tau, \quad 0 \leq t<T_{m}
$$

and

$$
E_{1}(0) \leq E_{0} e^{C t}, \quad 0 \leq t<T_{m} .
$$

From (26), we obtain $T_{m}=+\infty$. If the conclusion $T_{m}=+\infty$ is false, then $T_{m}<\infty$. By (26), we get

$$
E_{1}(t) \leq E_{1}(0) e^{C T_{m}}, \quad \text { for } 0 \leq t<T_{m},
$$

which contradicts (16).

Proof of Theorem 1.5 It follows from Corollary 2.7 that problem (5) admits a unique local solution $u \in C^{2}\left(\left[0, T_{m}\right] ; H^{3}\right)$ and $(-\Delta)^{-\frac{1}{2}} u_{t} \in C^{1}\left(\left[0, T_{m}\right) ; L^{2}\right)$.

Multiplying (22) by $-\Delta u_{t}$, we obtain

$$
\begin{aligned}
& \frac{1}{2} \frac{d}{d t}\left[\left\|\nabla u_{t}\right\|^{2}+\left\|\nabla^{3} u_{t}\right\|^{2}+\left\|\Delta u_{t}\right\|^{2}+\|\nabla u\|^{2}+\left\|\nabla^{3} u\right\|^{2}+\|\Delta u\|^{2}\right] \\
& \quad=-\left(f^{\prime}(u) \nabla u, \nabla^{3} u_{t}\right)+\left(\nabla u, \nabla u_{t}\right), \quad 0 \leq t<T_{m} .
\end{aligned}
$$

From (23) and (27), for $0 \leq t<T_{m}$, we get

$$
\begin{aligned}
\frac{1}{2} \frac{d}{d t}[ & {\left[\left\|u_{t}\right\|^{2}+2\left\|\nabla u_{t}\right\|^{2}+\left\|\Delta u_{t}\right\|^{2}+\left\|\nabla^{3} u_{t}\right\|^{2}\right.} \\
& \left.+\left\|\nabla^{3} u\right\|^{2}+\|u\|^{2}+\left\|\nabla^{3} u\right\|^{2}+2\|\Delta u\|^{2}\right] \\
= & \left(u, u_{t}\right)+\left(\nabla u, \nabla u_{t}\right)-\left(f^{\prime}(u) \nabla u, \nabla^{3} u_{t}\right)+\left(f^{\prime}(u) \nabla u, \nabla u_{t}\right)
\end{aligned}
$$


and

$$
\begin{aligned}
\left(u, u_{t}\right)+\left(\nabla u, \nabla u_{t}\right) \leq & \frac{1}{2}\left(\|u\|^{2}+\left\|u_{t}\right\|^{2}+\|\nabla u\|^{2}+\left\|\nabla u_{t}\right\|^{2}\right), \\
-\left(f^{\prime}(u) \nabla u, \nabla u_{t}\right) \leq & C\left(\left\|u_{t}\right\|^{2}+\left\|\nabla u_{t}\right\|^{2}+\left\|\Delta u_{t}\right\|^{2}\right. \\
& \left.+\|u\|^{2}+2\|\nabla u\|^{2}+2\|\Delta u\|^{2}\right), \\
\left(f^{\prime}(u) \nabla u, \nabla^{3} u_{t}\right) \leq & \left\|f^{\prime}(u)\right\|_{4}\|\nabla u\|_{4}\left\|\nabla^{3} u_{t}\right\| \leq C\|u\|_{H^{2}}\left\|\nabla^{3} u_{t}\right\| \\
\leq & C\left(\|u\|_{H^{2}}^{2}+\left\|\nabla^{3} u_{t}\right\|^{2}\right) \\
\leq & C\left(\|u\|^{2}+\|\nabla u\|^{2}+\|\Delta u\|^{2}+\left\|\nabla^{3} u_{t}\right\|^{3}\right) .
\end{aligned}
$$

Let

$$
\begin{aligned}
E_{2}(t)= & \frac{1}{2}\left(\left\|u_{t}\right\|^{2}+2\left\|\nabla u_{t}\right\|^{2}+\left\|\Delta u_{t}\right\|^{2}+\left\|\nabla^{3} u_{t}\right\|^{2}+\|u\|^{2}\right. \\
& \left.+2\|\nabla u\|^{2}+2\|\Delta u\|^{2}+\left\|\nabla^{3} u\right\|^{2}\right) .
\end{aligned}
$$

Using (28) and the estimates above, we obtain

$$
\frac{d}{d t} E_{2}(t) \leq C E_{2}(t), \quad E_{2}(t) \leq E_{2}(0)+C \int_{0}^{t} E_{2}(\tau) d \tau, \quad 0 \leq t<T_{m}
$$

and

$$
E_{2}(t) \leq E_{2}(0) e^{C t}, \quad 0 \leq t<T_{m} .
$$

Thus $T_{m}=+\infty$.

Proof of Theorem 1.6 From Corollary 2.7, it follows that problem (5) admits a unique local solution $u \in C^{2}\left(\left[0, T_{m}\right] ; H^{4}\right)$, with $(-\Delta)^{-\frac{1}{2}} u_{t} \in C^{1}\left(\left[0, T_{m}\right) ; L^{2}\right)$, where $T_{m}$ is the maximal existence time of $u$.

Multiplying (22) by $-\Delta^{2} u_{t}$ and integrating on $R^{n}$, we have

$$
\begin{aligned}
& \frac{1}{2} \frac{d}{d t}\left[\left\|\Delta u_{t}\right\|^{2}+\left\|\nabla^{3} u_{t}\right\|^{2}+\left\|\Delta^{2} u_{t}\right\|^{2}+\|\Delta u\|^{2}+\left\|\nabla^{3} u\right\|^{2}+\left\|\Delta^{2} u\right\|^{2}\right] \\
& \quad=\left(\Delta f(u), \Delta^{2} u_{t}\right)+\left(\Delta u, \Delta u_{t}\right) .
\end{aligned}
$$

From (23) and (30), we obtain

$$
\begin{aligned}
& \frac{1}{2} \frac{d}{d t}\left[\left\|u_{t}\right\|^{2}+2\left\|\Delta u_{t}\right\|^{2}+\left\|\nabla^{3} u_{t}\right\|^{2}+\left\|\Delta^{2} u_{t}\right\|^{2}+\|u\|^{2}\right. \\
& \left.+\|\nabla u\|^{2}+2\|\Delta u\|^{2}+2\left\|\nabla^{3} u\right\|^{2}+\left\|\Delta^{2} u\right\|^{2}\right] \\
& =\left(u, u_{t}\right)+\left(\Delta u, \Delta u_{t}\right)-\left(\Delta f(u), \Delta^{2} u_{t}\right)+\left(\Delta u, \Delta u_{t}\right), \quad 0 \leq t<T_{m}, \\
& \left(u, u_{t}\right)+\left(\Delta u, \Delta u_{t}\right) \leq \frac{1}{2}\left(\|u\|^{2}+\left\|u_{t}\right\|^{2}+\|\Delta u\|^{2}+\left\|\Delta u_{t}\right\|^{2}\right), \\
& -\left(f^{\prime}(u) \nabla u, \nabla u_{t}\right) \leq C\|u\|_{H^{2}}\left\|u_{t}\right\|_{H^{2}} \\
& \leq C\left(\|u\|^{2}+\|\nabla u\|^{2}+\|\Delta u\|^{2}+\left\|u_{t}\right\|^{2}+\left\|\Delta u_{t}\right\|^{2}\right),
\end{aligned}
$$




$$
\begin{aligned}
\left(\Delta f(u), \Delta^{2} u_{t}\right) & \leq\|\Delta f(u)\|\left\|\Delta^{2} u_{t}\right\| \\
& \leq C\left(\|u\|_{H^{2}}^{2}+\left\|\Delta^{2} u_{t}\right\|^{2}\right) \\
& \leq C\left(\|u\|^{2}+\|\nabla u\|^{2}+\|\Delta u\|^{2}+\left\|\Delta^{2} u_{t}\right\|^{2}\right) .
\end{aligned}
$$

Let

$$
\begin{aligned}
E_{3}(t)= & \frac{1}{2}\left(\left\|u_{t}\right\|^{2}+2\left\|\Delta u_{t}\right\|^{2}+\left\|\nabla^{3} u_{t}\right\|^{2}+\|u\|^{2}+\|\nabla u\|^{2}\right. \\
& \left.+2\|\Delta u\|^{2}+\left\|\nabla^{3} u\right\|^{2}+\left\|\Delta^{2} u\right\|^{2}\right) .
\end{aligned}
$$

Then, from (31) and the estimates above, we obtain

$$
\frac{d}{d t} E_{3}(t) \leq C E_{3}(t), \quad E_{3}(t) \leq E_{3}(0)+C \int_{0}^{t} E_{3}(\tau) d \tau, \quad 0 \leq t<T_{m}
$$

and

$$
E_{3}(t) \leq E_{3}(0) e^{C t}, \quad 0 \leq t<T_{m},
$$

from which one derives $T_{m}=+\infty$.

\section{Finite-time blow-up of the solution}

In this section, we study the finite-time blow-up of the solution for problem (5).

Lemma 5.1 Under the assumptions of Corollary 2.7, we have

$$
(-\Delta)^{-\frac{1}{2}} u \in C^{2}\left(\left[0, T_{m}\right) ; L^{2}\right)
$$

provided that $(-\Delta)^{-\frac{1}{2}} u_{0} \in L^{2}$.

Proof From $(-\Delta)^{-\frac{1}{2}} u \in C^{2}\left(\left[0, T_{m}\right) ; L^{2}\right)$ and

$$
(-\Delta)^{-\frac{1}{2}} u=(-\Delta)^{-\frac{1}{2}} u_{0}+\int_{0}^{t}(-\Delta)^{-\frac{1}{2}} u_{\tau} d \tau
$$

we obtain the result.

Proof of Theorem 1.7 Let $u \in C^{2}\left(\left[0, T_{m}\right) ; H^{s}\right)$ and $(-\Delta)^{-\frac{1}{2}} u_{t} \in C^{1}\left(\left[0, T_{m}\right) ; L^{2}\right)$ be the unique local solution of problem (5). Then, by Lemma 5.1 , we have $(-\Delta)^{-\frac{1}{2}} u \in C^{2}\left(\left[0, T_{m}\right) ; L^{2}\right)$, where $T_{m}$ is the maximal existence time of $u$. Suppose that $T_{m}=+\infty$. Then $u \in$ $C^{2}\left([0, \infty) ; H^{s}\right)$ with $(-\Delta)^{-\frac{1}{2}} u \in C^{2}\left([0, \infty) ; L^{2}\right)$ and $(-\Delta)^{-\frac{1}{2}} u_{t} \in C^{1}\left([0, \infty) ; L^{2}\right)$. Let

$$
H(t)=\left\|(-\Delta)^{-\frac{1}{2}} u\right\|^{2}+\|u\|^{2}+\|\nabla u\|^{2}, \quad 0 \leq t<\infty,
$$

then

$$
H^{\prime}(t)=2\left((-\Delta)^{-\frac{1}{2}} u_{t},(-\Delta)^{-\frac{1}{2}} u\right)+2\left(\nabla u_{t}, \nabla u\right)+2\left(u, u_{t}\right)
$$




$$
\begin{aligned}
H^{\prime \prime}(t)= & 2\left\|(-\Delta)^{-\frac{1}{2}} u_{t}\right\|^{2}+2\left\|\nabla u_{t}\right\|^{2}+2\left\|u_{t}\right\|^{2}+2\left((-\Delta)^{-\frac{1}{2}} u_{t t},(-\Delta)^{-\frac{1}{2}} u\right) \\
& +2\left(\nabla u_{t t}, \nabla u\right)+2\left(u_{t t}, u\right) \\
= & 2\left\|(-\Delta)^{-\frac{1}{2}} u_{t}\right\|^{2}+2\left\|\nabla u_{t}\right\|^{2}+2\left\|u_{t}\right\|^{2}+2\left((-\Delta)^{-1} u_{t t}, u\right) \\
& -2\left(\Delta u_{t t}, u\right)+2\left(u_{t t}, u\right) \\
= & 2\left\|(-\Delta)^{-\frac{1}{2}} u_{t}\right\|^{2}+2\left\|\nabla u_{t}\right\|^{2}+2\left\|u_{t}\right\|^{2}-2 I(u) .
\end{aligned}
$$

Using the energy equality (7), we obtain

$$
\frac{1}{2}\left\|(-\Delta)^{-\frac{1}{2}} u_{t}\right\|^{2}+\frac{1}{2}\left\|u_{t}\right\|^{2}+\frac{1}{2}\left\|\nabla u_{t}\right\|^{2}+\frac{1}{2}\|u\|_{H^{1}}^{2}+\int_{R^{n}} F(u) d x=E(0),
$$

from which one derives

$$
\frac{1}{2}\left\|(-\Delta)^{-\frac{1}{2}} u_{t}\right\|^{2}+\frac{1}{2}\left\|u_{t}\right\|^{2}+\frac{1}{2}\left\|\nabla u_{t}\right\|^{2}+\frac{p-1}{2(p+1)}\|u\|_{H^{1}}^{2}+\frac{1}{p+1} I(u)=E(0)
$$

and

$$
\begin{aligned}
-2 I(u)= & (p+1)\left[\left\|(-\Delta)^{-\frac{1}{2}} u_{t}\right\|^{2}+\left\|\nabla u_{t}\right\|^{2}+\left\|u_{t}\right\|^{2}\right] \\
& +(p-1)\|u\|_{H^{1}}^{2}-2(p+1) E(0) .
\end{aligned}
$$

Substituting (35) into (33), we obtain

$$
H^{\prime \prime}(t)=(p+3)\left[\left\|(-\Delta)^{-\frac{1}{2}} u_{t}\right\|^{2}+\left\|\nabla u_{t}\right\|^{2}+\left\|u_{t}\right\|^{2}\right]+(p-1)\|u\|_{H^{1}}^{2}-2(p+1) E(0) .
$$

On the other hand, from (33), we get

$$
\begin{aligned}
\left(H^{\prime}(t)\right)^{2}= & 4\left((-\Delta)^{-\frac{1}{2}} u_{t},(-\Delta)^{-\frac{1}{2}} u\right)+\left(\nabla u_{t}, \nabla u\right)+\left(u, u_{t}\right)^{2} \\
= & 4\left[\left((-\Delta)^{-\frac{1}{2}} u_{t},(-\Delta)^{-\frac{1}{2}} u\right)^{2}+\left(\nabla u_{t}, \nabla u\right)^{2}+\left(u, u_{t}\right)^{2}\right. \\
& +2\left((-\Delta)^{-\frac{1}{2}} u_{t},(-\Delta)^{-\frac{1}{2}} u\right)\left(\nabla u_{t}, \nabla u\right) \\
& \left.+2\left((-\Delta)^{-\frac{1}{2}} u_{t},(-\Delta)^{-\frac{1}{2}} u\right)\left(u, u_{t}\right)+2\left(\nabla u_{t}, \nabla u\right)\left(u, u_{t}\right)\right] \\
\leq & 4\left[\left\|(-\Delta)^{-\frac{1}{2}} u_{t}\right\|^{2}\left\|(-\Delta)^{-\frac{1}{2}} u\right\|^{2}+\left\|\nabla u_{t}\right\|^{2}\|\nabla u\|^{2}+\left\|u_{t}\right\|^{2}\|u\|^{2}\right. \\
& +\left\|(-\Delta)^{-\frac{1}{2}} u_{t}\right\|^{2}\|\nabla u\|^{2}+\left\|(-\Delta)^{-\frac{1}{2}} u\right\|^{2}\left\|\nabla u_{t}\right\|^{2}+\left\|(-\Delta)^{-\frac{1}{2}} u\right\|^{2}\|u\|^{2} \\
& \left.+\left\|(-\Delta)^{-\frac{1}{2}} u\right\|^{2}\left\|u_{t}\right\|^{2}+\left\|\nabla u_{t}\right\|^{2}\|u\|^{2}+\|\nabla u\|^{2}\left\|u_{t}\right\|^{2}\right] \\
= & 4 H(t)\left(\left\|(-\Delta)^{-\frac{1}{2}} u_{t}\right\|^{2}+\left\|\nabla u_{t}\right\|^{2}+\left\|u_{t}\right\|^{2}\right) .
\end{aligned}
$$

From (36) and (37), we get

$$
\begin{aligned}
H(t) H^{\prime \prime}(t)-\frac{p+3}{4}\left(H^{\prime}(t)\right)^{2} & \geq H(t)\left((p-1)\|u\|_{H^{1}}^{2}-2(p+1) E(0)\right) \\
& >H(t)\left((p-1)\|u\|_{H^{1}}^{2}-2(p+1) d\right) .
\end{aligned}
$$


Using $I\left(u_{0}\right)<0$ and Theorem 1.3, we get $u \in V_{2}$ and $I(u)<0$ for $0 \leq t \leq \infty$. Hence, by Lemma 3.3, we obtain $H(t)>0$ for $0 \leq t<\infty$. Using Lemma 3.5, we have $(p-1)\|u\|_{H^{1}}^{2}>$ $2(p+1) d$. Thus, we get

$$
H(t) H^{\prime \prime}(t)-\frac{p+3}{4}\left(H^{\prime}(t)\right)^{2}>0, \quad 0 \leq t<\infty .
$$

On the other hand, from (36), we obtain

$$
\begin{aligned}
H^{\prime \prime}(t) & \geq(p-1)\|u\|_{H^{1}}^{2}-2(p+1) E(0) \\
& =(p-1)\|u\|_{H^{1}}^{2}-2(p+1) d+2(p+1)(d-E(0)) \\
& >2(p+1)(d-E(0))=\delta_{0}, \quad 0 \leq t<\infty .
\end{aligned}
$$

Hence there exists a $t_{0} \geq 0$ such that $H^{\prime}(t)>0$, from which, together with $H\left(t_{0}\right)>0$ and (37), one derives that there exists a $T_{1}>0$ such that

$$
\lim _{t \rightarrow T_{1}} H(t)=\infty
$$

which contradicts $u \in C^{2}\left([0, \infty) ; H^{s}\right),(-\Delta)^{-\frac{1}{2}} u \in C^{2}\left([0, \infty) ; L^{2}\right)$. Finally, from $T_{m}<\infty$ and Corollary 2.4, we obtain

$$
\lim _{t \rightarrow T_{m}} \sup \left(\|u(t)\|_{H^{s}}+\left\|u_{t}(t)\right\|_{H^{s}}\right)=+\infty
$$

\section{Competing interests}

The author declares to have no competing interests.

\section{Author?s contributions}

The author declares to have read and approved the final manuscript.

\section{Acknowledgements}

The author would like to thank the editor and the reviewers for their constructive suggestions and helpful comments.

The work was partially supported by the National Natural Science Foundation of China (grant number 11101069).

Received: 10 September 2014 Accepted: 2 December 2014 Published online: 10 January 2015

\section{References}

1. Boussinesq, J: Théorie des ondes et de remous qui se propagent le long d? un canal rectangulaire horizontal, en communiquant au liquide contenu dans ce canal des vitesses sensiblement pareilles de la surface au fond. J. Math. Pures Appl. 17, 55-108 (1872)

2. Linares, F: Global existence of small solutions for a generalized Boussinesq equation. J. Differ. Equ. 106, 257-293 (1993)

3. Xue, R: Local and global existence of solutions for the Cauchy problem of a generalized Boussinesq equation. J. Math. Anal. Appl. 316, 307-327 (2006)

4. Yang, Z, Guo, B: Cauchy problem for the multi-dimensional Boussinesq type equation. J. Math. Anal. Appl. 340, 64-80 (2008)

5. Lai, SY, Wu, YH: The asymptotic solution of the Cauchy problem for a generalized Boussinesq equation. Discrete Contin. Dyn. Syst. 3, 401-408 (2003)

6. Lin, Q, Wu, YH, Loxton, R: On the Cauchy problem for a generalized Boussinesq equation. J. Math. Anal. Appl. 353, 186-195 (2009)

7. Liu, Y: Instability and blow-up of solutions to a generalized Boussinesq equation. SIAM J. Math. Anal. 26, 1527-1546 (1995)

8. Liu, Y: Decay and scattering of small solutions of a generalized Boussinesq equation. J. Funct. Anal. 147, 51-68 (1997)

9. Liu, YC, Xu, RZ: Global existence and blow up of solutions for Cauchy problem of generalized Boussinesq equation. Physica D 237, 721-731 (2008)

10. Xu, RY: Local and global existence of solutions for the Cauchy problem of a generalized Boussinesq equation. J. Math. Anal. Appl. 316, 307-327 (2006)

11. Chen, GW, Wang, YP, Wang, SB: Initial boundary value problem of the generalized cubic double dispersion equation. J. Math. Anal. Appl. 299, 563-577 (2004) 
12. Wang, SB, Chen, GW: Cauchy problem of the generalized double dispersion equation. Nonlinear Anal. 64, 159-173 (2006)

13. Liu, YC, Xu, RZ: Potential well method for Cauchy problem of the generalized double dispersion equations. J. Math. Anal. Appl. 338, 1169-1187 (2008)

14. Schneider, G, Eugene, CW: Kawahara dynamics in dispersive media. Physica D 152-153, 384-394 (2001)

15. Wang, Y, Mu, CL: Blow-up and scattering of solution for a generalized Boussinesq equation. Appl. Math. Comput. 188 1131-1141 (2007)

16. Wang, Y, Mu, CL: Global existence and blow-up of the solutions for the multidimensional generalized Boussinesq equation. Math. Methods Appl. Sci. 30, 1403-1417 (2007)

17. Wang, SB, Xu, GX: The Cauchy problem for the Rosenau equation. Nonlinear Anal. 71, 456-466 (2009)

18. Wang, HW, Wang, SB: Global existence and asymptotic behavior of solution for the Rosenau equation with hydrodynamical damped term. J. Math. Anal. Appl. 401, 763-773 (2013)

19. $\mathrm{Xu}, \mathrm{RZ}$, Liu, YC, Yu, T: Global existence of solution for Cauchy problem of multidimensional generalized double dispersion equation. Nonlinear Anal. 71, 4977-4983 (2009)

20. Xu, RZ, Liu, YC, Liu, BW: The Cauchy problem for a class of the multidimensional Boussinesq-type equation. Nonlinear Anal. 74, 2425-2437 (2011)

21. Wang, SB, Xue, HX: Global solution for a generalized Boussinesq equation. Appl. Math. Comput. 204, 130-136 (2008)

\section{Submit your manuscript to a SpringerOpen ${ }^{\circ}$ journal and benefit from:}

- Convenient online submission

Rigorous peer review

- Immediate publication on acceptance

- Open access: articles freely available online

- High visibility within the field

- Retaining the copyright to your article 